\title{
EVERY LINEAR THRESHOLD FUNCTION HAS A LOW-WEIGHT APPROXIMATOR
}

\author{
Rocco A. Servedio
}

\begin{abstract}
Given any linear threshold function $f$ on $n$ Boolean variables, we construct a linear threshold function $g$ which disagrees with $f$ on at most an $\epsilon$ fraction of inputs and has integer weights each of magnitude at most $\sqrt{n} \cdot 2^{\tilde{O}\left(1 / \epsilon^{2}\right)}$. We show that the construction is optimal in terms of its dependence on $n$ by proving a lower bound of $\Omega(\sqrt{n})$ on the weights required to approximate a particular linear threshold function. We give two applications. The first is a deterministic algorithm for approximately counting the fraction of satisfying assignments to an instance of the zero-one knapsack problem to within an additive $\pm \epsilon$. The algorithm runs in time polynomial in $n$ (but exponential in $1 / \epsilon^{2}$ ). In our second application, we show that any linear threshold function $f$ is specified to within error $\epsilon$ by estimates of its Chow parameters (degree 0 and 1 Fourier coefficients) which are accurate to within an additive $\pm 1 /\left(n \cdot 2^{\tilde{O}\left(1 / \epsilon^{2}\right)}\right)$. This is the first such accuracy bound which is inverse polynomial in $n$, and gives the first polynomial bound (in terms of $n$ ) on the number of examples required for learning linear threshold functions in the "restricted focus of attention" framework.
\end{abstract}

Keywords. Boolean functions, linear threshold functions, Chow parameters, computational learning theory.

Subject classification. 06E30, 52C07, 52C35, 68Q15, 68Q32.

\section{Introduction}

A linear threshold function, or LTF, is a Boolean function $f:\{-1,1\}^{n} \rightarrow$ $\{-1,1\}$ for which there exist $w=\left(w_{1}, \ldots, w_{n}\right) \in \mathbf{R}^{n}$ and $\theta \in \mathbf{R}$ such that

$$
f(x)=\operatorname{sgn}\left(\sum_{i=1}^{n} w_{i} x_{i}-\theta\right) \text { for all } x \in\{-1,1\}^{n} .
$$

Here the sgn function is defined as $\operatorname{sgn}(x)=1$ for $x \geq 0, \operatorname{sgn}(x)=-1$ for $x<0$. Linear threshold functions (sometimes referred to in the literature as "threshold gates" or "weighted threshold gates") have been extensively studied 
since the 1960s, see Dertouzos (1965); Hu (1965); Muroga (1971), and currently play an important role in many areas of theoretical computer science. In complexity theory, complexity classes of fundamental interest such as $\mathrm{TC}^{0}$ are defined in terms of linear threshold functions, and much effort has been expended on understanding the computational power of single linear threshold gates and shallow circuits composed of these gates, see, e.g., Goldmann et al. (1992); Goldmann \& Karpinski (1998); Hajnal et al. (1993); Hofmeister (1996); Orponen (1992); Razborov (1992). Linear threshold functions also play a central role in computational learning theory and machine learning; many of the most widely used and successful learning techniques such as support vector machines (Shawe-Taylor \& Cristianini 2000), various boosting algorithms (Freund 1995; Freund \& Schapire 1997), and fundamental algorithms such as Perceptron (Block 1962; Novikoff 1962) and Winnow (Littlestone 1988, 1991) are based on linear threshold functions in an essential way. Algorithms which learn linear threshold functions have also proved instrumental in the design of the fastest known learning algorithms for various expressive classes of Boolean functions (see, e.g., Klivans et al. 2004; Klivans \& Servedio 2001; Maass \& Turan 1994).

It is not hard to see that any linear threshold function $f:\{-1,1\}^{n} \rightarrow$ $\{-1,1\}$ has some representation - in fact infinitely many - in which all the weights $w_{i}$ are integers. It is of considerable interest in both learning theory and complexity theory (see the references cited above) to understand how large these integer weights must be. Easy counting arguments show that most linear threshold functions over $\{-1,1\}^{n}$ require integer weights of magnitude $2^{\Omega(n)}$. A classic result of Muroga et al. (1961) shows that any linear threshold function $f$ over $\{-1,1\}^{n}$ can be expressed using integer weights $w_{1}, \ldots, w_{n}$ each satisfying $\left|w_{i}\right| \leq 2^{O(n \log n)}$. (This result has since been rediscovered many times, see, e.g., Hong 1987; Raghavan 1988.) Håstad (1994) gave a matching lower bound by exhibiting a particular linear threshold function and proving that any integer representation for it must have weights of magnitude $2^{\Omega(n \log n)}$. Thus the size of weights that are required to (exactly) compute linear threshold functions is now rather well understood.

In this paper we are interested in the size of weights that are required to approximately compute linear threshold functions. Let us say that a Boolean function $g$ is an $\epsilon$-approximator for $f$ if $\operatorname{Pr}[g(x) \neq f(x)] \leq \epsilon$, where the probability is over a uniform choice of $x$ from $\{-1,1\}^{n}$. We consider the following:

Question: Let $f$ be an arbitrary linear threshold function. If $g$ is an LTF which $\epsilon$-approximates $f$ and has integer weights, how large do the weights of $g$ need to be? 
We feel that this is a natural question to investigate on its own merits; further motivation is given by the applications described in Section 1.2. As a first indication that the landscape can change dramatically when we switch from exact to approximate computation, consider the comparison function $\operatorname{COMP}(x, y)$ on $2 n$ bits which outputs 1 iff $x \geq y$ (viewing $x$ and $y$ as $n$ bit binary numbers). It is not hard to show that $\operatorname{COMP}(x, y)$ is a linear threshold function which requires integer weights of magnitude $2^{\Omega(n)}$, but it is also easy to see that $\operatorname{COMP}(x, y)$ is $\epsilon$-approximated by a linear threshold function which has only $2 \log (1 / \epsilon)$ many relevant variables and integer weights each at most $O(1 / \epsilon)$.

\subsection{Our results: approximating linear threshold functions using} small weights. We give a fairly complete answer to the above question. In Section 3 we prove a lower bound by exhibiting a simple linear threshold function $f$ and showing that any $\epsilon$-approximating linear threshold function for $f$ must have some weight of magnitude $\Omega(\sqrt{n})$. Perhaps surprisingly, we also show that $O(\sqrt{n})$ is an upper bound on the weights required to approximate any linear threshold function to any constant accuracy $\epsilon>0$. Our main result is the following, proved in Section 4:

TheOREM 1.1. Let $f:\{-1,1\}^{n} \rightarrow\{-1,1\}$ be any linear threshold function. For any $\epsilon>0$ there is a $\epsilon$-approximating LTF $g$ with integer weights $u_{1}, \ldots, u_{n}$ which satisfy

$$
\sum_{i=1}^{n} u_{i}^{2} \leq n \cdot 2^{\tilde{O}\left(1 / \epsilon^{2}\right)} .
$$

Theorem 1.1 immediately implies that each individual weight $u_{i}$ is at most $\sqrt{n} \cdot 2^{\tilde{O}\left(1 / \epsilon^{2}\right)}$ in magnitude. It also implies that the sum of the magnitudes of all $n$ weights is at most $n \cdot 2^{\tilde{O}\left(1 / \epsilon^{2}\right)}$.

In terms of the dependence on $\epsilon$, the "right" answer is somewhere between $(1 / \epsilon)^{\omega(1)}$ (see Section 7 ) and our upper bound of $2^{\tilde{O}\left(1 / \epsilon^{2}\right)}$ from Theorem 1.1; narrowing this gap is an interesting direction for future work.

1.2. Applications. We give two main applications of Theorem 1.1. The first, in Section 5, is a deterministic algorithm for approximately counting the fraction of satisfying assignments to any linear threshold function (or equivalently, counting the number of solutions to a zero-one knapsack problem) to within additive accuracy $\pm \epsilon$. The algorithm runs in time $\tilde{O}\left(n^{2} / \epsilon\right)+2^{\tilde{O}\left(1 / \epsilon^{2}\right)}$.

The second application is to the problem of reconstructing a linear threshold function from (approximations to) its low-degree Fourier coefficients. Various 
forms of this problem have been studied since the 1960s (see Birkendorf et al. 1998; Bruck 1990; Chow 1961; Goldberg 2001; Kaszerman 1963; Winder 1971; we give a detailed description of prior work in Section 6). We show that for any constant $\epsilon>0$, any linear threshold function $f$ is information-theoretically specified to within error $\epsilon$ by estimates of its degree- 0 and degree- 1 Fourier coefficients (sometimes known as its Chow parameters) which are accurate to within an additive $\pm 1 / O(n)$ :

Theorem 1.2. Let $f:\{-1,1\}^{n} \rightarrow\{-1,1\}$ be any linear threshold function. Let $g:\{-1,1\}^{n} \rightarrow\{-1,1\}$ be any Boolean function which satisfies

$$
|\hat{g}(S)-\hat{f}(S)| \leq 1 /\left(n \cdot 2^{\tilde{O}\left(1 / \epsilon^{2}\right)}\right)
$$

for each $S=\emptyset,\{1\},\{2\}, \ldots,\{n\}$. Then $\operatorname{Pr}[f(x) \neq g(x)] \leq \epsilon$.

This is the first known accuracy bound which is inverse polynomial in $n$; previous work of Goldberg (2001) gave a 1/quasipoly $(n)$ bound. We also observe that there is an easy $1 / \Omega(\sqrt{n})$ lower bound on the accuracy required. Theorem 1.2 directly yields the first polynomial bound (in terms of $n$ ) on the number of examples required for learning linear threshold functions in the "restricted focus of attention" learning framework of Ben-David \& Dichterman (1998).

1.3. Related work. To the best of our knowledge, ours is the first work to explicitly address the question of what weights are required to approximate linear threshold functions over the $n$-dimensional Boolean cube. A somewhat related problem was addressed in Servedio (2004), where it was shown that any monotone linear threshold function over the Boolean cube can be approximated to any constant accuracy by a monotone Boolean formula of polynomial size. Our proof of Theorem 1.1 proceeds by considering the same three cases that were considered in the proof of the main result of Servedio (2004) but the details are significantly different; see Section 4.1 for a detailed discussion.

\section{Preliminaries}

For $v \in \mathbf{R}^{n}$ we write $\|v\|$ to denote $\sqrt{v_{1}^{2}+\cdots+v_{n}^{2}}$. We write $u \cdot v$ to denote the inner product $\sum_{i=1}^{n} u_{i} v_{i}$ of two vectors $u, v \in \mathbf{R}^{n}$.

We will use standard tail bounds on sums of independent random variables, in particular the following form of the Hoeffding bound in which the deviation is bounded in terms of $\|w\|$. 
Theorem 2.1. Fix any $0 \neq w \in \mathbf{R}^{n}$. For any $\gamma>0$, we have

$$
\operatorname{Pr}_{x \in\{-1,1\}^{n}}[w \cdot x \geq \gamma\|w\|] \leq e^{-\gamma^{2} / 2} \text { and } \operatorname{Pr}_{x \in\{-1,1\}^{n}}[w \cdot x \leq-\gamma\|w\|] \leq e^{-\gamma^{2} / 2} .
$$

Another useful tool from probability theory is the following theorem, which upper bounds the probability mass that certain sums of independent random variables can have on any small region. The result can be derived from Theorem 2.14 in Petrov (1995); a short self-contained proof is given in Servedio (2004).

Theorem 2.2. Fix any $w \in \mathbf{R}^{n}$ with $\left|w_{i}\right| \leq 1$ for each $i$. Then for every $\lambda \geq 1$ and $\theta \in \mathbf{R}$, we have

$$
\operatorname{Pr}_{x \in\{-1,1\}^{n}}[|w \cdot x-\theta| \leq \lambda] \leq 6 \lambda /\|w\| .
$$

We use $\tilde{O}(\cdot)$ notation to hide polylogarithmic dependence on the argument of $\tilde{O}(\cdot)$; for instance, if $g(n)=n^{2} \log ^{3} n$ we may write $g(n)=\tilde{O}\left(n^{2}\right)$.

\section{The lower bound}

In this section we exhibit a linear threshold function $f$ and show that any representation with integer weights which computes a good approximator for $f$ must have some weight of magnitude $\Omega(\sqrt{n})$.

Let $f:\{-1,1\}^{n+1} \rightarrow\{-1,1\}$ be defined as

$$
f\left(x_{1}, \ldots, x_{n+1}\right)=\operatorname{sgn}\left(x_{1}+\cdots+x_{n}+n x_{n+1}-n\right) .
$$

Note that $f\left(x_{1}, \ldots, x_{n}, 1\right)=\operatorname{Maj}\left(x_{1}, \ldots, x_{n}\right)$ and $f\left(x_{1}, \ldots, x_{n},-1\right)=-1$ for all $x$. For convenience we assume that $n \equiv 2 \bmod 4$, but it will be clear that this assumption can be removed without loss of generality.

Our main result of this section is:

Theorem 3.1. Let $h:\{-1,1\}^{n+1} \rightarrow\{-1,1\}$ be any LTF which $1 / 10$-approximates $f$, and let $\operatorname{sgn}\left(v_{1} x_{1}+\cdots+v_{n+1} x_{n+1}-\theta\right)$ be any integer representation for $h$. Then $\left|v_{i}\right|=\Omega(\sqrt{n})$ for some $i$.

A straightforward application of the Hoeffding bound shows that for any $\epsilon=\Theta(1)$, there is indeed an $\epsilon$-approximating LTF $\operatorname{sgn}\left(x_{1}+\cdots+x_{n}+N x_{n+1}-N\right)$ for $f$ in which $N=\Theta(\sqrt{n})$. 
Proof of Theorem 3.1. Let $h_{1}$ denote the function $h\left(x_{1}, \ldots, x_{n}, 1\right)=$ $\operatorname{sgn}\left(v_{1} x_{1}+\cdots+v_{n} x_{n}+v_{n+1}-\theta\right)$. Since $h$ is an 1/10-approximator for $f$, we have that $\operatorname{Pr}_{x_{1}, \ldots, x_{n}}\left[h_{1}(x) \neq \operatorname{Maj}(x)\right] \leq 1 / 5$.

The following claim will be useful. (Stronger bounds could be given with more effort, but the $n / 2$ bound is good enough for our purposes and admits a very simple proof.)

Claim 3.2. The function $h_{1}$ must depend on at least $n / 2$ variables.

Proof. Suppose $h_{1}$ has $r<n / 2$ relevant variables; we will show that then $\operatorname{Pr}_{x_{1}, \ldots, x_{n}}\left[h_{1} \neq \mathrm{Maj}\right]>1 / 5$. For each $\ell=1, \ldots, n$ let $g_{\ell}:\{-1,1\}^{\ell} \rightarrow\{-1,1\}$ be the Boolean function on variables $x_{1}, \ldots, x_{\ell}$ which is the closest approximator to $\operatorname{Maj}\left(x_{1}, \ldots, x_{n}\right)$. It follows that

$$
\operatorname{Pr}\left[h_{1} \neq \mathrm{Maj}\right] \geq \operatorname{Pr}\left[g_{r} \neq \mathrm{Maj}\right] \geq \operatorname{Pr}\left[g_{n / 2} \neq \mathrm{Maj}\right] .
$$

It is easy to see that each function $g_{\ell}$ is simply $\operatorname{Maj}\left(x_{1}, \ldots, x_{\ell}\right)$. (On each input $x=\left(x_{1}, \ldots, x_{\ell}\right)$, the value of $g_{\ell}$ is the bit $b \in\{-1,1\}$ such that the majority of the $2^{n-\ell}$ extensions $\left(x_{1}, \ldots, x_{n}\right)$ of $x$ have $\operatorname{Maj}\left(x_{1}, \ldots, x_{n}\right)=b$; it is easy to check that this bit $b$ is $\operatorname{Maj}\left(x_{1}, \ldots, x_{\ell}\right)$.) We thus have

$$
\begin{aligned}
\operatorname{Pr}\left[g_{n / 2} \neq \operatorname{Maj}\right]= & \operatorname{Pr}\left[\operatorname{Maj}\left(x_{1}, \ldots, x_{n / 2}\right) \neq \operatorname{Maj}\left(x_{1}, \ldots, x_{n}\right)\right] \\
\geq & \operatorname{Pr}\left[\operatorname{sgn}\left(x_{n / 2+1}+\cdots+x_{n}\right) \neq \operatorname{sgn}\left(x_{1}+\cdots+x_{n / 2}\right)\right. \\
& \left.\&\left|x_{n / 2+1}+\cdots+x_{n}\right|>\left|x_{1}+\cdots+x_{n / 2}\right|\right] \\
= & \operatorname{Pr}\left[\operatorname{sgn}\left(x_{n / 2+1}+\cdots+x_{n}\right) \neq \operatorname{sgn}\left(x_{1}+\cdots+x_{n / 2}\right)\right] \\
& \times \operatorname{Pr}\left[\left|x_{n / 2+1}+\cdots+x_{n}\right|>\left|x_{1}+\cdots+x_{n / 2}\right|\right] \\
\geq & (1 / 2)(1 / 2-o(1))>1 / 5
\end{aligned}
$$

where the second equality holds because the signs and magnitudes of the sums are independent (since $n / 2$ is odd, each sign is achieved with probability exactly $1 / 2)$.

By Claim 3.2 we may assume without any loss of generality that each of $x_{1}, \ldots, x_{n / 2}$ is a relevant variable for $h_{1}$. Since each $v_{i}$ is an integer, it follows that each of $\left|v_{1}\right|, \ldots,\left|v_{n / 2}\right|$ is at least 1 . Letting $v^{\prime}$ denote the $n$-dimensional vector $\left(v_{1}, \ldots, v_{n}\right)$, we have that $\left\|v^{\prime}\right\| \geq \sqrt{n / 2}$.

Since $h_{1}$ is a $1 / 5$-approximator to $\operatorname{Maj}\left(x_{1}, \ldots, x_{n}\right)$ and $\operatorname{Pr}[\operatorname{Maj}(x)=1]=$ $1 / 2-o(1)$, we have that $\operatorname{Pr}_{x_{1}, \ldots, x_{n}}\left[v_{1} x_{1}+\cdots+v_{n} x_{n}+v_{n+1} \geq \theta\right] \geq 0.295$. Similarly, since $h_{-1}(x) \stackrel{\text { def }}{=} \operatorname{sgn}\left(v_{1} x_{1}+\cdots+v_{n} x_{n}-v_{n+1}-\theta\right)$ is a $1 / 5$-approximator to the 
constant function -1 , it must be the case that $\operatorname{Pr}_{x_{1}, \ldots, x_{n}}\left[v_{1} x_{1}+\cdots+v_{n} x_{n}-v_{n+1} \geq\right.$ $\theta] \leq 0.2$. These two inequalities imply that $v_{n+1}>0$ and that

$$
\underset{x_{1}, \ldots, x_{n}}{\operatorname{Pr}}\left[\left|v_{1} x_{1}+\cdots+v_{n} x_{n}-\theta\right| \leq v_{n+1}\right] \geq 0.095 .
$$

Let $v_{\max }$ denote $\max _{i=1, \ldots, n}\left|v_{i}\right|$, let $u_{i}=v_{i} / v_{\max }$ for $i=1, \ldots, n$, and let $\lambda=v_{n+1} / v_{\max }$. Suppose first that $\lambda \geq 1$. In this case we can apply Theorem 2.2 to obtain

$$
0.095 \leq(3.3)=\operatorname{Pr}\left[\left|u \cdot x-\theta / v_{\max }\right| \leq \lambda\right] \leq \frac{6 \lambda}{\|u\|}=\frac{6 \lambda v_{\max }}{\left\|v^{\prime}\right\|}=\frac{6 v_{n+1}}{\left\|v^{\prime}\right\|}
$$

which implies that $v_{n+1}=\Omega(\sqrt{n})$. On the other hand, if $\lambda<1$ then $v_{n+1}$ is not the largest weight; it is not difficult to show that such a linear threshold function must have large error with respect to $f$. Alternately, we can observe that if $\lambda<1$ then again by Theorem 2.2, we have

$0.095 \leq(3.3)=\operatorname{Pr}\left[\left|u \cdot x-\theta / v_{\max }\right| \leq \lambda\right] \leq \operatorname{Pr}\left[\left|u \cdot x-\theta / v_{\max }\right| \leq 1\right] \leq \frac{6}{\|u\|}=\frac{6 v_{\max }}{\left\|v^{\prime}\right\|}$

which implies $v_{\max }=\Omega(\sqrt{n})$. So in each case some weight is $\Omega(\sqrt{n})$, and Theorem 3.1 is proved.

\section{Proof of Theorem 1.1}

Let $\epsilon>0$ be given and let $f:\{-1,1\}^{n} \rightarrow\{-1,1\}$ be any linear threshold function. Without loss of generality we may suppose that $f(x)=\operatorname{sgn}\left(\sum_{i=1}^{n} w_{i} x_{i}-\theta\right)$ where we have $1=\left|w_{1}\right| \geq\left|w_{2}\right| \geq \cdots \geq\left|w_{n}\right|>0$.

As in the argument of Servedio (2004) we consider different cases depending on the value of $\|w\|$. In each case we show how to construct an $\epsilon$-approximating LTF with integer weights that satisfy the claimed bound.

Case I: $\|w\| \geq 12 / \epsilon$. Very roughly speaking, the idea of this case is that many of the weights are "large" compared with the largest weight $w_{1}$. (For intuition, consider the majority function in which $1=w_{1}=\cdots=w_{n}$; this function has the largest possible value of $\|w\|$.) In this case the construction works by rounding the weights to a carefully chosen granularity and showing that this only incurs small error. We actually prove a stronger version of Theorem 1.1 in this case by showing that the sum of squared weights for the $\epsilon$-approximator is at most $O\left(n \ln (1 / \epsilon) / \epsilon^{2}\right)$ rather than $n \cdot 2^{\tilde{O}\left(1 / \epsilon^{2}\right)}$. 
Let

$$
\alpha=\frac{\epsilon\|w\|}{6 \sqrt{2 n \ln (4 / \epsilon)}} .
$$

For each $i=1, \ldots, n$ let $u_{i}$ be the value obtained by rounding $w_{i}$ to the nearest integer multiple of $\alpha$. Let $g(x)=\operatorname{sgn}\left(\sum_{i=1}^{n} u_{i} x_{i}-\theta\right)$, or equivalently $g(x)=$ $\operatorname{sgn}\left(\sum_{i=1}^{n}\left(u_{i} / \alpha\right) x_{i}-\theta / \alpha\right)$. We will prove the following lemma:

LEMma 4.1. The linear threshold function $g(x)=\operatorname{sgn}\left(\sum_{i=1}^{n}\left(u_{i} / \alpha\right) x_{i}-\theta / \alpha\right)$ is an $\epsilon$-approximator for $f$ with integer weights each at most $O(\sqrt{n \ln (1 / \epsilon)})$ in magnitude. Moreover, the sum of squares of weights is $O\left(n \ln (1 / \epsilon) / \epsilon^{2}\right)$.

Proof. For $i=1, \ldots, n$ let $e_{i}=w_{i}-u_{i}$, so $u \cdot x=w \cdot x-e \cdot x$. Let $\lambda \geq 1$ be such that

$$
\frac{\epsilon}{2}=\frac{6 \lambda}{\|w\|}
$$

We have that $\operatorname{sgn}(u \cdot x-\theta) \neq \operatorname{sgn}(w \cdot x-\theta)$ only if either $|e \cdot x| \geq \lambda$ or $|w \cdot x-\theta| \leq \lambda$. We will show that each of these two events occurs with probability at most $\epsilon / 2$ for a random $x$, and consequently $\operatorname{Pr}[g(x) \neq f(x)] \leq \epsilon$.

First we bound $\operatorname{Pr}[|e \cdot x| \geq \lambda]$. We have that $\left|e_{i}\right| \leq \frac{1}{2} \alpha$ for each $i$, so the vector $e=\left(e_{1}, \ldots, e_{n}\right)$ has $\|e\| \leq \frac{1}{2} \alpha \sqrt{n}$. Observing that $\lambda=\sqrt{2 \ln (4 / \epsilon)} \cdot \frac{1}{2} \alpha \sqrt{n}$, the Hoeffding bound (Theorem 2.1) gives

$$
\operatorname{Pr}[|e \cdot x| \geq \lambda] \leq \operatorname{Pr}[|e \cdot x| \geq \sqrt{2 \ln (4 / \epsilon)} \cdot\|e\|] \leq 2 e^{-\left(\sqrt{2 \ln (4 / \epsilon)}^{2} / 2\right.}=\epsilon / 2 .
$$

To bound $\operatorname{Pr}[|w \cdot x-\theta| \leq \lambda]$ we simply apply Theorem 2.2 ; this gives us $\operatorname{Pr}[|w \cdot x-\theta| \leq \lambda] \leq 6 \lambda /\|w\|$, which equals $\epsilon / 2$ by our original condition on $w$ in Case I.

Thus far we have shown that $g$ is an $\epsilon$-approximating LTF for $f$. It is clear that $g$ has a representation with integer weights each at most $1 / \alpha=$ $O\left(\frac{\sqrt{n \ln (1 / \epsilon)}}{\|w\| \epsilon}\right)=O(\sqrt{n \ln (1 / \epsilon)})$, where the second equality uses $\epsilon\|w\| \geq 12$. In fact we can bound the magnitude of the sum of squares of these integer weights. Let

$$
v_{i}=u_{i} / \alpha
$$

so each $v_{i}$ is an integer and $g(x)=\operatorname{sgn}(v \cdot x-\theta / \alpha)$. Rounding each weight $w_{i}$ to obtain $u_{i}$ is easily seen to increase its magnitude by at most a factor of two. Consequently we have that each $\left|v_{i}\right| \leq 2\left|w_{i}\right| / \alpha$, and so we have

$$
\sum_{i=1}^{n} v_{i}^{2} \leq 4\left(\sum_{i=1}^{n} w_{i}^{2}\right) / \alpha^{2}=4\|w\|^{2} \cdot \frac{72 n \ln (4 / \epsilon)}{\epsilon^{2}\|w\|^{2}}=O\left(n \ln (1 / \epsilon) / \epsilon^{2}\right) .
$$


Case II: $\|w\|<12 / \epsilon$. Note that since $\left|w_{1}\right|=1$, this inequality can be rewritten as $w_{1}^{2} /\left(\sum_{j=1}^{n} w_{j}^{2}\right)>\epsilon^{2} / 144$.

Let us set up some notation. We let

$C_{1}=4 \ln (4 / \epsilon), \quad C_{2}=72 \ln \left(2 C_{1} / \epsilon\right), \quad \tau=\epsilon^{2} / 144$, and $\ell=\frac{3}{\tau} \ln \left(C_{2} / \tau\right) \ln (4 / \epsilon)$.

Note that $\ell=\tilde{O}\left(1 / \epsilon^{2}\right)$. We assume that $\ell \leq n$; observe that if this is not the case, then $n=\tilde{O}\left(1 / \epsilon^{2}\right)$, hence Theorem 1.1 follows trivially from the standard $2^{O(n \log n)}$ weight upper bound of Muroga et al..

As in Servedio (2004), we consider two subcases.

Case IIa: $w_{k}^{2} /\left(\sum_{j=k}^{n} w_{j}^{2}\right)>\epsilon^{2} / 144$ for all $k=1, \ldots, \ell$. This case can be thought of as capturing those $w$ 's for which the first $\ell$ weights decrease quite rapidly, e.g., in geometric progression. (For intuition one can consider the ODDMAXBIT function, i.e., a decision list with alternating output bits; it is straightforward to check that for the standard linear threshold function representation of this function, the value of $\|w\|$ is an absolute constant independent of $n$, and a bound on successive weights similar to that of Case IIa indeed holds.)

In this case, instead of rounding the weights $w_{i}$ as we did in Case I, we will simply truncate the linear threshold function after the first $\ell$ variables and show that the resulting LTF is an $\epsilon$-approximator for $f$. Since this truncated LTF depends on only $\ell$ variables, the standard upper bound of Muroga et al. implies that it has an integer representation with each weight at most $2^{O(\ell \log \ell)}$ and hence sum of squared weights also $2^{O(\ell \log \ell)}=2^{\tilde{O}\left(1 / \epsilon^{2}\right)}$.

Let $g(x)=\operatorname{sgn}\left(w_{1} x_{1}+\cdots+w_{\ell} x_{\ell}-\theta\right)$. Let

$$
W=w_{\ell+1}^{2}+\cdots+w_{n}^{2},
$$

and let

$$
\eta=\sqrt{2 W \ln (4 / \epsilon)} .
$$

We have that $g(x) \neq f(x)$ only if either $\left|w_{\ell+1} x_{\ell+1}+\cdots+w_{n} x_{n}\right| \geq \eta$ or $\mid w_{1} x_{1}+$ $\cdots+w_{\ell} x_{\ell}-\theta \mid \leq \eta$. We will show that these events each have probability at most $\epsilon / 2$ and thus obtain $\operatorname{Pr}[g(x) \neq f(x)] \leq \epsilon$.

Bounding the first probability is easy; by our choice of $\eta$, the Hoeffding bound gives

$$
\operatorname{Pr}\left[\left|w_{\ell+1} x_{\ell+1}+\cdots+w_{n} x_{n}\right| \geq \eta\right] \leq 2 e^{-2 \ln (4 / \epsilon) / 2}=\epsilon / 2 .
$$

We now show that $\operatorname{Pr}\left[\left|w_{1} x_{1}+\cdots+w_{\ell} x_{\ell}-\theta\right| \leq \eta\right] \leq \epsilon / 2$. Note that since we are in Case IIa, we have $w_{\ell}^{2}>\left(\epsilon^{2} / 144\right) \sum_{j=\ell+1}^{n} w_{j}^{2}$ and thus $w_{\ell}>(\epsilon / 12) \sqrt{W}=$ 
$(\epsilon / 12)(\eta / \sqrt{2 \ln (4 / \epsilon)})$. It therefore suffices to show that

$$
\operatorname{Pr}\left[\left|w_{1} x_{1}+\cdots+w_{\ell} x_{\ell}-\theta\right| \leq(12 / \epsilon) \sqrt{2 \ln (4 / \epsilon)} w_{\ell}\right] \leq \epsilon / 2 .
$$

For $i=1, \ldots, n$ we will write $W_{i}$ to denote $\sum_{j=i}^{n} w_{j}^{2}$; note that $W_{i}=$ $w_{i}^{2}+W_{i+1}$. The following lemma will be useful (recall that $\tau=\epsilon^{2} / 144$ ):

Lemma 4.5. For $a<b \leq \ell$, we have $W_{b}<(1-\tau)^{b-a} W_{a}<\frac{(1-\tau)^{b-a}}{\tau} w_{a}^{2}$.

Proof. Since we are in Case IIa we have $w_{a}^{2}>\tau W_{a}=\tau w_{a}^{2}+\tau W_{a+1}$, or equivalently $(1-\tau) w_{a}^{2}>\tau W_{a+1}$. Adding $(1-\tau) W_{a+1}$ to both sides gives $(1-$ $\tau)\left(w_{a}^{2}+W_{a+1}\right)=(1-\tau) W_{a}>W_{a+1}$. This implies that $W_{b}<(1-\tau)^{b-a} W_{a}$; the second inequality follows from $w_{a}^{2}>\tau W_{a}$.

We divide the weights $w_{1}, \ldots, w_{\ell}$ into blocks of consecutive weights as follows. The first block $B_{1}$ is $\left\{w_{1}, \ldots, w_{k_{1}}\right\}$ where $k_{1}$ is the first index such that $W_{k_{1}+1}<w_{1}^{2} / C_{2}$. (Recall that $C_{2}=72 \ln \left(2 C_{1} / \epsilon\right)$.) Similarly, the $i$-th block $B_{i}$ is $\left\{w_{k_{i-1}+1}, \ldots, w_{k_{i}}\right\}$ where $k_{i}$ is the first index such that $W_{k_{i}+1}<w_{k_{i-1}+1}^{2} / C_{2}$.

Corollary 4.6. Each block $B_{i}$ is of length at most $\frac{1}{\tau} \ln \left(C_{2} / \tau\right)$.

Proof. By Lemma 4.5, the length $\left|B_{i}\right|$ of the $i$-th block must satisfy $1 / C_{2} \leq$ $(1-\tau)^{\left|B_{i}\right|} / \tau$; the corollary follows from this.

Recalling that $\ell=\frac{3}{\tau} \ln \left(C_{2} / \tau\right) \ln (4 / \epsilon)$, we have that there are at least $3 \ln (4 / \epsilon)$ many blocks of weights in $w_{1}, \ldots, w_{\ell}$.

Let us view the choice of a uniform $\left(x_{1}, \ldots, x_{\ell}\right) \in\{-1,1\}^{\ell}$ as taking place in successive stages, where in the $i$-th stage the variables corresponding to the $i$-th block $B_{i}$ are chosen. The rest of our analysis in Case IIa will only deal with the first $\ln (4 / \epsilon)$ blocks so for the rest of Case IIa we assume that $i \leq \ln (4 / \epsilon)$.

Immediately after the $i$-th stage, some value - call it $\xi_{i}$ - has been determined for $w_{1} x_{1}+\cdots+w_{k_{i}} x_{k_{i}}$. The following lemma shows that if $\xi_{i}$ is too far from $\theta$, then it is unlikely that the remaining variables $x_{k_{i}+1}, \ldots, x_{\ell}$ will come out in such a way as to make the final sum sufficiently close to $\theta$. (In the following lemma, recall that $C_{1}=4 \ln (4 / \epsilon)$.)

LEMma 4.7. If $\left|\xi_{i}-\theta\right| \geq 2 \sqrt{W_{k_{i}+1}} \sqrt{2 \ln \left(2 C_{1} / \epsilon\right)}$, then we have

$$
\operatorname{Pr}_{x_{k_{i}+1}, \ldots, x_{\ell}}\left[\left|w_{1} x_{1}+\cdots+w_{\ell} x_{\ell}-\theta\right| \leq(12 / \epsilon) \sqrt{2 \ln (4 / \epsilon)} w_{\ell}\right] \leq \epsilon / C_{1} .
$$


Proof. By the lower bound on $\left|\xi_{i}-\theta\right|$ in the hypothesis of the lemma, it can only be the case that $\left|w_{1} x_{1}+\cdots+w_{\ell} x_{\ell}-\theta\right| \leq(12 / \epsilon) \sqrt{2 \ln (4 / \epsilon)} w_{\ell}$ if

$$
\left|w_{k_{i}+1} x_{k_{i}+1}+\cdots+w_{\ell} x_{\ell}\right| \geq 2 \sqrt{W_{k_{i}+1}} \sqrt{2 \ln \left(2 C_{1} / \epsilon\right)}-(12 / \epsilon) \sqrt{2 \ln (4 / \epsilon)} w_{\ell}
$$

Since $i \leq \ln (4 / \epsilon)$ and each block is of length at most $\frac{1}{\tau} \ln \left(C_{2} / \tau\right)$ by Corollary 4.6, we have that $k_{i}+1 \leq \frac{1}{\tau} \ln \left(C_{2} / \tau\right) \ln (4 / \epsilon)+1$. Recalling the definition of $\ell$, it follows that $\left(\ell-\left(k_{i}+1\right)\right) / 2>\frac{1}{\tau} \ln (12 / \epsilon)$. Now using Lemma 4.5, we have that

$$
w_{\ell} \leq \sqrt{W_{\ell}} \leq(1-\tau)^{\left(\ell-\left(k_{i}+1\right)\right) / 2} \sqrt{W_{k_{i}+1}} \leq \frac{\epsilon}{12} \sqrt{W_{k_{i}+1}} .
$$

Rearranging this inequality and using $2 C_{1} \geq 4$, it follows that the RHS of (4.9) is at least $\sqrt{2 \ln \left(2 C_{1} / \epsilon\right)} \cdot \sqrt{W_{k_{i}+1}}$. So to prove the lemma it suffices to bound $\operatorname{Pr}_{x_{k_{i}+1}, \ldots, x_{\ell}}\left[\left|w_{k_{i}+1} x_{k_{i}+1}+\cdots+w_{\ell} x_{\ell}\right| \geq \sqrt{2 \ln \left(2 C_{1} / \epsilon\right)} \cdot \sqrt{W_{k_{i}+1}}\right]$ by $\epsilon / C_{1}$. But since $w_{k_{i}+1}^{2}+\cdots+w_{\ell}^{2} \leq W_{k_{i}+1}$, the Hoeffding bound implies that this probability is at most $2 e^{-\left(\sqrt{2 \ln \left(2 C_{1} / \epsilon\right)}\right)^{2} / 2}=\epsilon / C_{1}$.

We now show that regardless of the value $\xi_{i-1}$ immediately before the $i$-th stage, immediately after the $i$-th stage it must be the case that $\left|\xi_{i}-\theta\right| \leq$ $2 \sqrt{W_{k_{i}+1}} \sqrt{2 \ln \left(2 C_{1} / \epsilon\right)}$ holds with probability at most $1 / 2$ over the choice of values for variables in block $B_{i}$ in the $i$-th stage.

Lemma 4.10. For any $\xi_{i-1} \in \mathbf{R}$, we have

$$
\operatorname{Pr}_{x_{k_{i-1}+1}, \ldots, x_{k_{i}}}\left[\left|\xi_{i}-\theta\right| \leq 2 \sqrt{W_{k_{i}+1}} \sqrt{2 \ln \left(2 C_{1} / \epsilon\right)}\right] \leq 1 / 2 .
$$

Proof. Since $\xi_{i}$ equals $\xi_{i-1}+\left(w_{k_{i-1}+1} x_{k_{i-1}+1}+\cdots+w_{k_{i}} x_{k_{i}}\right)$, we have $\left|\xi_{i}-\theta\right| \leq$ $2 \sqrt{W_{k_{i}+1}} \sqrt{2 \ln \left(2 C_{1} / \epsilon\right)}$ if and only if the value $w_{k_{i-1}+1} x_{k_{i-1}+1}+\cdots+w_{k_{i}} x_{k_{i}}$ lies in the interval

$\left[I_{L}, I_{R}\right]:=\left[\theta-\xi_{i-1}-2 \sqrt{W_{k_{i}+1}} \sqrt{2 \ln \left(2 C_{1} / \epsilon\right)}, \theta-\xi_{i-1}+2 \sqrt{W_{k_{i}+1}} \sqrt{2 \ln \left(2 C_{1} / \epsilon\right)}\right]$

of width $4 \sqrt{W_{k_{i}+1}} \sqrt{2 \ln \left(2 C_{1} / \epsilon\right)}$.

First suppose that $0 \notin\left[I_{L}, I_{R}\right]$, i.e., the whole interval has the same sign. If this is the case then $\operatorname{Pr}\left[w_{k_{i-1}+1} x_{k_{i-1}+1}+\cdots+w_{k_{i}} x_{k_{i}} \in\left[I_{L}, I_{R}\right]\right] \leq 1 / 2$ since by symmetry the value $w_{k_{i-1}+1} x_{k_{i-1}+1}+\cdots+w_{k_{i}} x_{k_{i}}$ is equally likely to be positive or negative.

Now suppose that $0 \in\left[I_{L}, I_{R}\right]$. By definition of $k_{i}$, we know that $\sqrt{W_{k_{i}+1}} \leq$ $\left|w_{k_{i-1}+1}\right| / \sqrt{C_{2}}$, and consequently we have that the width of the interval $\left[I_{L}, I_{R}\right]$ 
is at most $4\left|w_{k_{i-1}+1}\right| \sqrt{2 \ln \left(2 C_{1} / \epsilon\right)} / \sqrt{C_{2}}$, which is at most $\frac{2}{3}\left|w_{k_{i-1}+1}\right|$ by the definition of $C_{2}$. But now observe that once the value of $x_{k_{i-1}+1}$ is set to either +1 or -1 , this effectively shifts the "target interval," which now $w_{k_{i-1}+2} x_{k_{i-1}+2}+\cdots+$ $w_{k_{i}} x_{k_{i}}$ must hit, by a displacement of $w_{k_{i-1}+1}$ to become $\left[I_{L}-w_{k_{i-1}+1} x_{k_{i-1}+1}\right.$, $\left.I_{R}-w_{k_{i-1}+1} x_{k_{i-1}+1}\right]$. Since the original interval $\left[I_{L}, I_{R}\right]$ contained 0 and was of length at most $\frac{2}{3}\left|w_{k_{i-1}+1}\right|$, the new interval does not contain 0 , and thus again by symmetry we have that the probability (now over the choice of $x_{k_{i-1}+2}, \ldots, x_{k_{i}}$ ) that $w_{k_{i-1}+1} x_{k_{i-1}+1}+\cdots+w_{k_{i}} x_{k_{i}}$ lies in $\left.\left[I_{L}, I_{R}\right]\right]$ is at most $1 / 2$.

In order to have $\left|w_{1} x_{1}+\cdots+w_{\ell} x_{\ell}-\theta\right| \leq(12 / \epsilon) \sqrt{2 \ln (4 / \epsilon)} w_{\ell}$, it must be the case that either

(1) each $\left|\xi_{i}-\theta\right|<2 \sqrt{W_{k_{i}+1}} \sqrt{2 \ln \left(2 C_{1} / \epsilon\right)}$ for $i=1, \ldots, \ln (4 / \epsilon)$; or

(2) for some $i \leq \ln (4 / \epsilon)$ we have $\left|\xi_{i}-\theta\right| \geq 2 \sqrt{W_{k_{i}+1}} \sqrt{2 \ln \left(2 C_{1} / \epsilon\right)}$ but nonetheless $\left|w_{1} x_{1}+\cdots+w_{\ell} x_{\ell}-\theta\right|<(12 / \epsilon) \sqrt{2 \ln (4 / \epsilon)} w_{\ell}$.

Lemma 4.10 gives us that the probability of $(1)$ is at most $(1 / 2)^{\ln (4 / \epsilon)}=$ $\epsilon / 4$, and Lemma 4.7 gives us that the probability of (2) is at most $\ln (4 / \epsilon)$. $\epsilon / C_{1}=\epsilon / 4$. Thus the overall probability that $\left|w_{1} x_{1}+\cdots+w_{\ell} x_{\ell}-\theta\right| \leq$ $(12 / \epsilon) \sqrt{2 \ln (4 / \epsilon)} w_{\ell}$ is at most $\epsilon / 2$, and (4.4) is proved.

Case IIb: $w_{k}^{2} /\left(\sum_{j=k}^{n} w_{j}^{2}\right) \leq \epsilon^{2} / 144$ for some $k \in\{1, \ldots, \ell\}$. Roughly speaking, in this case the first $k-1$ weights decrease quite rapidly, but then the rate of decrease slows and $w_{k}$ is "not too large" compared with $w_{k+1}, \ldots, w_{n}$. It does not seem that we can simply truncate the weights $w_{k}, \ldots, w_{n}$ in this case; instead we round the weights $w_{k}, \ldots, w_{n}$ to obtain an $\epsilon / 2$-approximating LTF in which these weights are small integers. We then argue that this LTF is itself $\epsilon / 2$-close to an LTF with all small integer weights.

We define weight vectors $u^{\prime}, v^{\prime} \in \mathbf{R}^{n}$ as follows: For $i=1, \ldots, k-1$ let

$$
u_{i}^{\prime}=w_{i} /\left|w_{k}\right| .
$$

For $i=k, \ldots, n$ let $u_{i}^{\prime}$ be the value obtained by rounding $w_{i} /\left|w_{k}\right|$ to the nearest integer multiple of

$$
\alpha^{\prime} \stackrel{\text { def }}{=} \frac{(\epsilon / 2) \sqrt{w_{k}^{2}+\cdots+w_{n}^{2}}}{6\left|w_{k}\right| \sqrt{2 n \ln (8 / \epsilon)}} .
$$

(Note that everywhere $\alpha$ in Case I had an $\epsilon$, now $\alpha^{\prime}$ has $\epsilon / 2$.) Let

$$
v_{i}^{\prime}=u_{i}^{\prime} / \alpha^{\prime}
$$


for all $i=1, \ldots, n$. Finally let

$$
\theta^{\prime}=\theta /\left|w_{k}\right|
$$

and let $g:\{-1,1\}^{n} \rightarrow\{-1,1\}$ be the LTF

$$
g(x)=\operatorname{sgn}\left(u^{\prime} \cdot x-\theta^{\prime}\right)
$$

or equivalently $g(x)=\operatorname{sgn}\left(v^{\prime} \cdot x-\theta^{\prime} / \alpha^{\prime}\right)$.

We first show that $g$ is an $\epsilon / 2$-approximator for $f$ which has "almost all" small integer weights.

Lemma 4.11. The linear threshold function $g(x)=\operatorname{sgn}\left(v^{\prime} \cdot x-\theta^{\prime} / \alpha^{\prime}\right)$ is an $\epsilon / 2$-approximator for $f$. Each weight $v_{i}^{\prime}$ for $i \geq k$ is an integer of magnitude $O(\sqrt{n \ln (1 / \epsilon)})$, and we have $\sum_{i=k}^{n}\left(v_{i}^{\prime}\right)^{2}=O\left(n \ln (1 / \epsilon) / \epsilon^{2}\right)$.

Proof. Fix any setting $x_{1}^{*}, \ldots, x_{k-1}^{*}$ of the first $k-1$ bits. Let $f_{*}$ be the linear threshold function on $n-k+1$ variables which is obtained by fixing the first $k-1$ inputs of $f$ to $x_{1}^{*}, \ldots, x_{k-1}^{*}$; note that we may write $f_{*}\left(x_{k}, \ldots, x_{n}\right)$ as $\operatorname{sgn}\left(\sum_{j=k}^{n}\left(w_{j} /\left|w_{k}\right|\right) x_{j}-\theta^{\prime}+\sum_{j=1}^{k-1}\left(w_{j} /\left|w_{k}\right|\right) x_{j}^{*}\right)$. Similarly, let $g_{*}$ be the LTF on $n-k+1$ variables obtained by fixing the first $k-1$ inputs of $g$ to to $x_{1}^{*}, \ldots, x_{k-1}^{*}$, i.e.,

$$
g_{*}\left(x_{k}, \ldots, x_{n}\right)=\operatorname{sgn}\left(\sum_{j=k}^{n} v_{j}^{\prime} x_{j}-\theta^{\prime} / \alpha^{\prime}+\sum_{j=1}^{k-1} v_{j}^{\prime} x_{j}^{*}\right) .
$$

We have that $1=\left|w_{k} /\right| w_{k}|| \geq\left|w_{k+1} /\right| w_{k}|| \geq \cdots \geq\left|w_{n} /\right| w_{k}||>0$. Moreover, each weight $v_{i}^{\prime}$ for $i \geq k$ is obtained from $w_{i} /\left|w_{k}\right|$ by rounding to the nearest integer multiple of $\alpha^{\prime}$ (and then scaling by $\alpha^{\prime}$ to get integer weights). Since the thresholds of $f_{*}$ and $g_{*}$ match up as well (taking into account the scaling by $\alpha^{\prime}$ ), we may apply Lemma 4.1 , and conclude that $\operatorname{Pr}_{x_{k}, \ldots, x_{n}}\left[g_{*} \neq\right.$ $\left.f_{*}\right] \leq \epsilon / 2$. Since this holds for every restriction $x^{*} \in\{-1,1\}^{k-1}$, it follows that $\operatorname{Pr}_{x \in\{-1,1\}^{n}}[g(x) \neq f(x)] \leq \frac{\epsilon}{2}$. The claimed bounds on the weights $v_{i}^{\prime}$ for $i \geq k$ follow from Lemma 4.1 .

We next show that any linear threshold function which has "almost all" its weights integers whose sum of squares is small (such as $g$ ) can be $\epsilon / 2$ approximated by a linear threshold function with small integer weights. To do this we will need the following claim, the proof of which is deferred until later: 
Claim 4.12. Fix an integer $R>0$. Let $\Omega$ denote $\{-1,1\}^{k-1} \times\{-R,-R+$ $1, \ldots, R-1, R\}$. Let $h$ be any linear threshold function over $\Omega$, i.e., for some $w \in \mathbf{R}^{k}$ and $\theta \in \mathbf{R}$ we have that $h(x)=\operatorname{sgn}(w \cdot x-\theta)$ for all $x \in \Omega$. Then there is a representation of $h$ as $h(x)=\operatorname{sgn}(u \cdot x-\theta)$ in which

(a) each $u_{i}$ is an integer, and

(b) $\left|u_{i}\right| \leq R \cdot(k+1)$ ! for $i=1, \ldots, k-1$ and $\left|u_{k}\right| \leq(k+1)$ !.

This claim is an extension of Muroga et al.'s classic upper bound on the size of integer weights that are required to express linear threshold functions over the usual domain $\{-1,1\}^{n}$; we defer its proof until later.

LEMMA 4.13. Let $g:\{-1,1\}^{n} \rightarrow\{-1,1\}: g(x)=\operatorname{sgn}(s \cdot x-\mu)$ be a linear threshold function where $s_{k}, s_{k+1}, \ldots, s_{n}$ are all integers with $\sum_{j=k}^{n} s_{j}^{2} \leq N$. Then there is a linear threshold function $g^{\prime}(x)=\operatorname{sgn}(t \cdot x-\nu)$ which is an $\epsilon / 2$-approximator of $g$, where

(i) each $t_{i}$ is an integer;

(ii) $\left|t_{i}\right| \leq \sqrt{N \ln (1 / \epsilon)} \cdot 2^{O(k \log k)}$ for $i \leq k-1$; and

(iii) $\sum_{i=1}^{n} t_{i}^{2} \leq N \cdot \ln (1 / \epsilon) \cdot 2^{O(k \log k)}$.

Proof of Lemma 4.13. We first observe that by the Hoeffding bound, we have

$$
\operatorname{Pr}_{x_{k}, \ldots, x_{n}}\left[\left|s_{k} x_{k}+\cdots+s_{n} x_{n}\right|>\sqrt{2 \ln (4 / \epsilon)} \sqrt{N}\right] \leq 2 e^{-(\sqrt{2 \ln (4 / \epsilon)})^{2} / 2}=\epsilon / 2 .
$$

Intuitively, we can thus pretend that $\sum_{j=k}^{n} s_{k} x_{k}$ always has magnitude at most $\sqrt{2 \ln (4 / \epsilon)} \sqrt{N}$ and this causes us to incur error at most $\epsilon / 2$ (we will make this more precise later).

Now the pieces are in place to prove Lemma 4.13. Let

$$
R=\sqrt{2 \ln (4 / \epsilon)} \sqrt{N} .
$$

Given the LTF $g(x)=\operatorname{sgn}(s \cdot x-\mu)$, let $h: \Omega \rightarrow\{-1,1\}$ be the LTF

$$
h(x)=\operatorname{sgn}\left(\sum_{j=1}^{k-1} s_{i} x_{i}+x_{k}-\mu\right) .
$$


By Claim 4.12, we have that over the domain $\Omega, h$ is equivalent to $h(x)=$ $\operatorname{sgn}\left(\sum_{j=1}^{k} u_{i} x_{i}-\mu\right)$, where $u_{1}, \ldots, u_{k}$ satisfy conditions (a) and (b). Now consider $g^{\prime}:\{-1,1\}^{n} \rightarrow\{-1,1\}$,

$$
g^{\prime}(x)=\operatorname{sgn}\left(\sum_{i=1}^{k-1} u_{i} x_{i}+u_{k}\left(\sum_{j=k}^{n} s_{j} x_{j}\right)-\mu\right) .
$$

By our observation at the start of the proof, at least a $1-\epsilon / 2$ fraction of all $x \in\{-1,1\}^{n}$ have $\left|\sum_{j=k}^{n} s_{j} x_{j}\right| \leq R$. For each such $x$ we have $g^{\prime}(x)=$ $h\left(x_{1}, \ldots, x_{k-1}, \sum_{j=k}^{n} s_{j} x_{j}\right)=g(x)$. Thus $g^{\prime}$ is an $\epsilon / 2$-approximator of $g$ with integer weights $t_{1}, \ldots, t_{n}$, where $t_{i}=u_{i}$ for $i \leq k-1$ and $t_{j}=u_{k} s_{j}$ for $j \geq k$. Plugging in the bounds on $u_{i}, u_{k}, s_{j}$ from the conditions of Lemma 4.13 and Claim 4.12, the proof of Lemma 4.13 is done.

Combining Lemma 4.11 and Lemma 4.13, recalling that $k \leq \ell=\tilde{O}\left(1 / \epsilon^{2}\right)$, and taking $N$ in Lemma 4.13 to be $O\left(n \ln (1 / \epsilon) / \epsilon^{2}\right)$, we obtain the desired conclusion of Theorem 1.1 in Case IIb. It remains only to prove Claim 4.12.

Proof of Claim 4.12. We need only slightly modify known proofs of Muroga et al.'s upper bound for LTF weights over $\{-1,1\}^{n}$. In particular we closely follow the outline of the proof in Section 3 of Håstad (1994).

Let $H_{0}: \mathbf{R}^{k} \rightarrow \mathbf{R}$ be a linear function $H_{0}(x)=a \cdot x+t$ which satisfies the following conditions:

1. $\operatorname{sgn}\left(H_{0}(x)\right)=h(x)$ for each $x \in \Omega$.

2. $\left|H_{0}(x)\right| \geq 1$ for each $x \in \Omega$.

3. Among all linear functions which satisfy conditions (1) and (2) above, $H_{0}$ maximizes the number of $x \in \Omega$ which have $\left|H_{0}(x)\right|=1$. If there is more than one possible $H_{0}$ which achieves the maximum number, choose one arbitrarily.

Observe that since $h(x)$ is a linear threshold function over $\Omega$, there exists some linear function satisfying (1) and (2), and thus there does exist some $H_{0}$ satisfying (1)-(3) above.

As in Håstad (1994), let $x^{(1)}, \ldots, x^{(r)}$ be the set of points in $\Omega$ with $\mid H_{0}\left(x^{(i)} \mid=1\right.$. The argument in Håstad (1994) now directly implies that $H_{0}$ is uniquely determined by the equations

$$
H_{0}\left(x^{(i)}\right)=h\left(x^{(i)}\right) \quad \text { for } \quad i=1, \ldots, r .
$$


Consequently the coefficients $a_{1}, \ldots, a_{k}, t$ of $H_{0}(x)$ can be obtained by solving a linear system of $k+1$ equations:

$$
a_{1} x_{1}^{(i)}+\cdots+a_{k} x_{k}^{(i)}+t=h\left(x^{(i)}\right) \quad \text { for } \quad i=1, \ldots, k+1 .
$$

For each of these equations the right-hand side is \pm 1 as are the first $k-1$ coefficients $x_{1}^{(i)}, \ldots, x_{k-1}^{(i)}$ (and the coefficient of $t$ ), whereas the $k$-th coefficient $x_{k}^{(i)}$ is an integer in $\{-R, \ldots, R\}$.

Cramer's rule now tells us that for $j=1, \ldots, k$, we have

$$
a_{j}=\operatorname{det}\left(M_{j}\right) / \operatorname{det}(M)
$$

for suitable $(k+1) \times(k+1)$ matrices $M_{1}, \ldots, M_{k}, M$. More precisely, the matrix $M$ has as its $i$-th row the vector $x^{(i)}$ with a 1 appended as the $(k+1)$ st entry, and the matrix $M_{j}$ is $M$ but with the $j$-th column replaced by the column vector whose $i$-th entry is $h\left(x^{(i)}\right)$. Since all entries of $M$ except for the $k$-th column are \pm 1 and each element in the $k$-th column is an integer of magnitude at most $R$, we have that $\operatorname{det}(M)$ is an integer of magnitude at most $(k+1) ! R$, and the same is true for $\operatorname{det}\left(M_{1}\right), \ldots, \operatorname{det}\left(M_{k-1}\right)$. The matrix $M_{k}$ is a \pm 1 matrix so it satisfies $\left|\operatorname{det}\left(M_{k}\right)\right| \leq(k+1)$ !. Now since each of $a_{1}, \ldots, a_{k}$ has the same denominator we may clear it throughout and obtain a linear threshold function for $h$ whose $k$ integer weights are $\operatorname{det}\left(M_{1}\right), \ldots, \operatorname{det}\left(M_{k}\right)$. This concludes the proof of Claim 4.12 .

4.1. Discussion and consequences for monotone formula construction. The main result of Servedio (2004) is a proof that any monotone linear threshold function $f$ can be $\epsilon$-approximated by a monotone Boolean AND/OR formula of size $n^{10.6} \cdot 2^{\tilde{O}\left(1 / \epsilon^{4}\right)}$. The high-level structure of our proof of Theorem 1.1 is similar to that of Servedio (2004) in that the same cases I, IIa and IIb are considered, ${ }^{1}$ but there are some significant differences. First, in Case I of Servedio (2004) the weights are simply rounded to the nearest multiple of $1 / n$ rather than the nearest $\alpha=\frac{1}{O(\sqrt{n})}$ (ignoring the dependence on $\epsilon$ ). Second, our Case IIa is handled using a simpler argument in Servedio (2004) which only yields $\ell=\tilde{O}\left(1 / \epsilon^{4}\right)$ in Servedio (2004) rather than the $\ell=\tilde{O}\left(1 / \epsilon^{2}\right)$ we achieve here. Finally, since the goal in Servedio (2004) is to construct a monotone formula rather than a low-weight linear threshold function, a different approach is used in that paper to handle Case IIb. (In particular, a recursive tree-based

\footnotetext{
${ }^{1}$ Readers familiar with Servedio (2004) will note that Case IIa of this paper is Case IIb of Servedio (2004) and vice versa.
} 
decomposition is used in Servedio (2004) which yields a Boolean formula but not a linear threshold function.)

We observe that our new analysis of Case I and our new bound on $\ell$ can be straightforwardly worked into the arguments of Servedio (2004) to obtain a quantitative improvement of its main result: for $f:\{-1,1\}^{n} \rightarrow\{-1,1\}$ any monotone linear threshold function, there is a monotone Boolean formula of size $n^{5.3} \cdot 2^{\tilde{O}\left(1 / \epsilon^{2}\right)}$ which is an $\epsilon$-approximator for $f$. Briefly, the improvement from $n^{10.6}$ to $n^{5.3}$ comes from the fact that now in Case I, we have that the sum $\sum_{i=1}^{n}\left|v_{i}\right|$ of the integer weights of $g(x)$ is $O(n)$ rather than the $O\left(n^{2}\right)$ bound obtained in Servedio (2004) by rounding each weight to the nearest $1 / n$. This $O(n)$ is then plugged into Valiant's probabilistic construction (Valiant 1984) of monotone formulas of size $O\left(n^{5.3}\right)$ for the majority function on $n$ variables. We omit the details to avoid unnecessary repetition of Servedio (2004).

\section{Application to deterministic approximate counting}

We describe an application of our approach to the problem of approximately counting solutions of the zero-one knapsack problem. In an instance of zero-one knapsack we are given a vector $a=\left(a_{1}, \ldots, a_{n}\right) \in \mathbf{R}^{n}$ and a threshold $\theta \in \mathbf{R}$; the goal is to approximately compute the fraction $p$ of points $x \in\{0,1\}^{n}$ which satisfy the linear threshold function $\operatorname{sgn}\left(\sum_{i=1}^{n} a_{i} x_{i}-\theta\right)$. It is not hard to see that we may equivalently consider the domain of the LTF to be $\{-1,1\}^{n}$ as we have been doing throughout this paper.

The problem of efficiently computing a multiplicative $(1 \pm \epsilon)$-approximation of $p$ has received much attention (Dyer et al. 1993; Jerrum \& Sinclair 1997; Kannan 1994); the first polynomial-time algorithm was given by Morris \& Sinclair (1999) using sophisticated Monte Carlo Markov Chain techniques, and more recently a simpler randomized algorithm based on dynamic programming and "dart throwing" was given by Dyer (2003).

Our techniques, combined with the dynamic programming idea of Dyer (2003), give a simple deterministic algorithm for computing an $\epsilon$-accurate additive approximation of $p$. (Achieving such an additive approximation is trivial, of course, if randomization is allowed: simply make $O\left(1 / \epsilon^{2}\right)$ random draws from $\{-1,1\}^{n}$ and output the fraction of satisfying assignments in this sample as an approximation of $p$.) See Trevisan (2004) for work in a similar spirit on deterministically counting the fraction of satisfying assignments to a $k$-DNF to additive accuracy $\pm \epsilon$. (It should be noted, though, that for $k$-DNF an additive approximation to the fraction of satisfying assignments is sufficient to yield a multiplicative approximation, see the reduction given in Luby \& Velick- 
ovic (1996); no such reduction is known for the problem of counting satisfying assignments for linear threshold functions.)

ThEOREM 5.1. There is a deterministic $\tilde{O}\left(n^{2} / \epsilon\right)+2^{\tilde{O}\left(1 / \epsilon^{2}\right)}$-time algorithm with the following property: given an instance of the zero-one knapsack problem for which the true fraction of satisfying assignments in $\{-1,1\}^{n}$ is $p$, the algorithm outputs a value $\tilde{p}$ such that $|p-\tilde{p}| \leq \epsilon$.

Proof. Given $w_{1}, \ldots, w_{n}, \theta$, the high-level idea is to efficiently construct a linear threshold function $g(x)$ which $\epsilon$-approximates $f(x)=\operatorname{sgn}(w \cdot x-\theta)$ as in the proof of Theorem 1.1, and then use dynamic programming to exactly count the number of satisfying assignments to $g$.

Suppose first that $w_{1}, \ldots, w_{n}$ satisfy Case I of Section 4 . Then as in that section we round each weight to the nearest integer multiple of $\alpha$ and divide by $\alpha$ throughout to obtain an $\epsilon$-approximating linear threshold function $g(x)=\operatorname{sgn}\left(v \cdot x-\theta^{\prime}\right)$ with integer weights $v_{i}$ that satisfy $\sum_{i=1}^{n}\left|v_{i}\right| \leq M=$ $O\left(n \ln (1 / \epsilon) / \epsilon^{2}\right)$. Let

$$
F(r, s)=\left|\left\{x \in\{-1,1\}^{r}: \sum_{i=1}^{r} v_{i} x_{i}=s\right\}\right| .
$$

We can compute $F(r, s)$ for all $1 \leq r \leq n,-M \leq s \leq M$ in $O(n M)$ time with dynamic programming, using the initial condition $F(0,0)=1$ and the relation

$$
F(r+1, s)=F\left(r, s-v_{r+1}\right)+F\left(r, s+v_{r+1}\right) .
$$

The number of satisfying assignments to $g$ is $\sum_{s \geq \theta^{\prime}} F(n, s)$.

Now suppose that $w_{1}, \ldots, w_{n}$ satisfy Case IIa. In this case, we shall take $g(x)=\operatorname{sgn}\left(w_{1} x_{1}+\cdots+w_{\ell} x_{\ell}-\theta\right)$ to be the truncated LTF analyzed in Case IIb. Since this function depends only on $\ell$ inputs, we can go over all possible settings of the relevant variables and easily determine the number of satisfying assignments to $g$ in time $2^{O(\ell)}=2^{\tilde{O}\left(1 / \epsilon^{2}\right)}$.

Finally suppose that $w_{1}, \ldots, w_{n}$ satisfy Case IIb. In this case we use the linear threshold function $g(x)=\operatorname{sgn}\left(v^{\prime} \cdot x-\theta^{\prime} / \alpha^{\prime}\right)$ described in Lemma 4.11. This function $g$ has at most $k-1 \leq \ell$ weights which are not integers, and the integer weights have total magnitude bounded by $M=O\left(n \ln (1 / \epsilon) / \epsilon^{2}\right)$. So we can do dynamic programming as in Case I in $O(n M)$ time to compute the values of $F(n, s)$ as $s$ ranges over all integers between $-M$ and $M$. In an additional $O(M)$ time we can compute values $Z(t) \stackrel{\text { def }}{=} \sum_{s \geq t} F(n, t)$ for all $t$ between $-M$ and $M$. Given these values for $Z(t)$, we we can go over all (at 
most $2^{\ell}=2^{\tilde{O}\left(1 / \epsilon^{2}\right)}$ many) settings of the non-integer weights as in Case IIa and easily compute the total number of assignments that satisfy $g$.

\section{Approximating an LTF from noisy versions of its low-degree Fourier coefficients}

Recall that for a Boolean function $f:\{-1,1\}^{n} \rightarrow\{-1,1\}$, the Fourier coefficients $\{\hat{f}(S)\}_{S \subseteq[n]}$ of $f$ are the coefficients of the (unique) multilinear polynomial

$$
f(x)=\sum_{S \subseteq[n]} \hat{f}(S) x_{S} \quad \text { where } x_{S} \text { denotes } \prod_{i \in S} x_{i}
$$

which agrees with $f$ everywhere on $\{-1,1\}^{n}$. The degree of a Fourier coefficient $\hat{f}(S)$ is the degree $|S|$ of of the corresponding monomial.

The main result of this section is Theorem 1.2:

TheOREm 1.2 (RESTATED). Let $f:\{-1,1\}^{n} \rightarrow\{-1,1\}$ be any linear threshold function. Let $g:\{-1,1\}^{n} \rightarrow\{-1,1\}$ be any Boolean function which satisfies

$$
|\hat{g}(S)-\hat{f}(S)| \leq 1 /\left(n \cdot 2^{\tilde{O}\left(1 / \epsilon^{2}\right)}\right)
$$

for each $S=\emptyset,\{1\},\{2\}, \ldots,\{n\}$. Then $\operatorname{Pr}[f(x) \neq g(x)] \leq \epsilon$.

Chow (1961) proved that every linear threshold function is uniquely specified (among all Boolean functions) by its $n+1$ Fourier coefficients of degree 0 and 1 ; these coefficients are sometimes referred to as the Chow parameters of $f$. Following this result (which was later generalized by Bruck 1990), there has been interest in how to algorithmically obtain a weights-based representation $f(x)=\operatorname{sgn}(w \cdot x-\theta)$ of $f$ from its Chow parameters, see, e.g., Kaszerman (1963); Winder (1971). This seems to be a difficult problem, and we do not address it here.

A related question which has also been studied is the following: suppose we are given noisy rather than exact values of the Chow parameters. How does this affect the precision with which $f$ is (information-theoretically) specified by these parameters? One motivation for studying this question comes from the "1-restricted focus of attention" model in computational learning theory; roughly speaking this is a learning model in which the learner is only allowed to see a single bit $x_{i}$ of each example $x=\left(x_{1}, \ldots, x_{n}\right)$ used for learning (see Ben-David \& Dichterman 1994, 1998 for details). As observed by Birkendorf et al. (1998); Goldberg (2001), the class of linear threshold functions over $\{-1,1\}^{n}$ is uniform-distribution information-theoretically learnable from 
$\operatorname{poly}(n)$ many examples in this framework if and only if any linear threshold function is information-theoretically specified to high accuracy from Chow parameter estimates which are accurate to an additive $\pm 1 / \operatorname{poly}(n)$.

With this motivation Birkendorf et al. gave the following result:

THEOREM 6.1 (Birkendorf et al. 1998). Let $f(x)=\operatorname{sgn}\left(w_{1} x_{1}+\cdots+w_{n} x_{n}-\theta\right)$ be a linear threshold function with integer weights $w_{i}$ such that $W=\sum_{i=1}^{n}\left|w_{i}\right|$. Let $g:\{-1,1\}^{n} \rightarrow\{-1,1\}$ be any Boolean function which satisfies

$$
|\hat{g}(S)-\hat{f}(S)| \leq \frac{\epsilon}{W}
$$

for each $S=\emptyset,\{1\},\{2\}, \ldots,\{n\}$. Then $\operatorname{Pr}[f(x) \neq g(x)] \leq \epsilon$.

Theorem 6.1 gives a strong bound on the precision required in the Chow parameters if $f$ has low weight, but a weak bound for arbitrary LTFs since $W$ may need to be $2^{\Omega(n \log n)}$. Subsequently Goldberg (2001) gave an incomparable result which can be rephrased as follows:

TheOREM 6.2 (Goldberg 2001). Let $f$ be any linear threshold function, and let $g:\{-1,1\}^{n} \rightarrow\{-1,1\}$ be any Boolean function which satisfies

$$
|\hat{g}(S)-\hat{f}(S)| \leq(\epsilon / n)^{O(\log (n / \epsilon) \log (1 / \epsilon))}
$$

for each $S=\emptyset,\{1\},\{2\}, \ldots,\{n\}$. Then $\operatorname{Pr}[f(x) \neq g(x)] \leq \epsilon$.

In contrast, our bound in Theorem 1.2 has a worse dependence on $\epsilon$ but has a $1 / n$ rather than $1 /$ quasipoly $(n)$ dependence on $n$. Theorem 1.2 yields an affirmative answer (at least for constant $\epsilon$ ) to the open question of whether arbitrary linear threshold functions can be learned in the uniform distribution 1-RFA model with polynomial sample complexity:

Corollary 6.3. Fix any constant $\epsilon>0$. There is an algorithm for learning arbitrary linear threshold functions to accuracy $\epsilon$ under the uniform distribution in the 1-restricted focus of attention model, using poly $(n)$ many examples.

6.1. Proof of Theorem 1.2. Let $\epsilon>0$ be given and let $f:\{-1,1\}^{n} \rightarrow$ $\{-1,1\}$ be any linear threshold function. We may suppose that

$$
f(x)=\operatorname{sgn}(F(x)) \quad \text { where } \quad F(x)=\sum_{i=1}^{n} w_{i} x_{i}-\theta
$$

with $1=\left|w_{1}\right| \geq\left|w_{2}\right| \geq \cdots \geq\left|w_{n}\right| \geq 0$. Note that without loss of generality we have $|\theta| \leq \sum_{i=1}^{n}\left|w_{i}\right|$. 
Fix any $g:\{-1,1\}^{n} \rightarrow\{-1,1\}$ where for $S=\emptyset,\{1\}, \ldots,\{n\}$ we have $|\hat{g}(S)-\hat{f}(S)| \leq 1 / M$ with $M=n \cdot 2^{\tilde{O}\left(1 / \epsilon^{2}\right)}$. Let $D$ denote

$$
D \stackrel{\text { def }}{=}\left\{x \in\{-1,1\}^{n}: g(x) \neq f(x)\right\}
$$

and $\tau$ denote $|D| / 2^{n}$. We will show that $\tau \leq \epsilon$ and thus establish Theorem 1.2.

We have

$$
\begin{aligned}
\mathbf{E}[|F(x)|] & =\mathbf{E}[f F]=\sum_{S \subseteq[n]} \hat{f}(S) \hat{F}(S) \\
& =\sum_{|S| \leq 1} \hat{f}(S) \hat{F}(S)=-\hat{f}(\emptyset) \theta+\sum_{i=1}^{n} \hat{f}(\{i\}) w_{i} \\
& \leq \hat{g}(\emptyset)(-\theta)+\hat{g}(\{1\}) w_{1}+\cdots+\hat{g}(\{n\}) w_{n}+\left(|\theta|+\sum_{i=1}^{n}\left|w_{i}\right|\right) / M
\end{aligned}
$$

The second equality above is Parseval's identity, the third is because F's only nonzero Fourier coefficients are of degree 0 and 1 , and the fourth is by definition of $F$. The inequality above is from our assumption on the Fourier coefficients of $g$. Using Parseval again and writing $B$ to denote $\left(|\theta|+\sum_{i=1}^{n}\left|w_{i}\right|\right) / M$, we have that the right-hand side of the above inequality equals

$$
\sum_{|S| \leq 1} \hat{g}(S) \hat{F}(S)+B=\sum_{S \subseteq[n]} \hat{g}(S) \hat{F}(S)+B=\mathbf{E}[g(x) F(x)]+B .
$$

Rearranging, this gives

$$
\left(|\theta|+\sum_{i=1}^{n}\left|w_{i}\right|\right) / M \geq \mathbf{E}[|F(x)|-g(x) F(x)]=\frac{2}{2^{n}} \sum_{x \in D}|F(x)| .
$$

Thus far we have followed the proof from Birkendorf et al. (1998) (which is itself closely based on Bruck 1990), and indeed it is not difficult to complete the proof of Theorem 6.1 from here. Instead we will use our ideas from Section 4 . The approach is to show that only a small number of points in $\{-1,1\}^{n}$ can have $|F(x)|$ very small, and thus if $|D|$ is large then the right hand side of (6.4) must be fairly large, which contradicts (6.4).

Case I: $\|w\| \geq 12 / \epsilon$. Let $\lambda \geq 1$ be such that

$$
\frac{\epsilon}{2}=\frac{6 \lambda}{\|w\|} .
$$


By Theorem 2.2 we have $\operatorname{Pr}[|F(x)| \leq \lambda] \leq \epsilon / 2$. Now suppose that $\tau>\epsilon$; this would mean that for at least $\frac{\epsilon}{2} 2^{n}$ points $x \in D$ we have $|F(x)|>\lambda=\epsilon\|w\| / 12$. But the bound (6.4) now gives

$$
\left(|\theta|+\sum_{i=1}^{n}\left|w_{i}\right|\right) / M \geq \frac{2}{2^{n}} \cdot \frac{\epsilon}{2} 2^{n} \cdot \frac{\epsilon\|w\|}{12}=\frac{\epsilon^{2}\|w\|}{12}
$$

This implies that we must have

$$
M \leq \frac{12\left(|\theta|+\sum\left|w_{i}\right|\right)}{\epsilon^{2}\|w\|} \leq \frac{\left(|\theta|+\sum\left|w_{i}\right|\right)}{\epsilon} \leq \frac{2 n}{\epsilon} .
$$

which contradicts the definition of $M$; so case I is proved.

Case II: $\|w\|<12 / \epsilon$. In this case we will use the following result due to Håstad (2005), which gives a bound on the rate at which weights need to decrease (from largest to smallest in magnitude) for any linear threshold function over $\{-1,1\}^{n}$.

Theorem 6.5 (Håstad 2005). Let $f:\{-1,1\}^{n} \rightarrow\{-1,1\}$ be any linear threshold function which depends on all $n$ variables. There is a representation $\operatorname{sgn}\left(\sum_{i} w_{i} x_{i}-\theta\right)$ for $f$ which is such that (assuming the weights $w_{1}, \ldots, w_{n}$ are ordered by decreasing magnitude $\left|w_{1}\right| \geq\left|w_{2}\right| \geq \cdots \geq\left|w_{n}\right|$ ) we have $\left|w_{i}\right| \geq \frac{\left|w_{1}\right|}{i !(n+1)}$ for all $i=2, \ldots, n$.

We prove Theorem 6.5 in Section 6.2. Note that this implies in general that for any constant $c=O(1)$, the $c$-th largest weight of any LTF need be at most $1 / O(n)$ times smaller than the largest weight. More specifically, in our context Theorem 6.5 lets us assume without loss of generality that the original weights $w_{1}, \ldots, w_{n}$ for $f$ satisfy $\left|w_{i}\right| \geq \frac{1}{i !(n+1)}$ for each $i$ (where we have $\left|w_{1}\right| \leq 1$ ). This will prove useful in both cases IIla and IIb below.

In the following $\ell=\tilde{O}\left(1 / \epsilon^{2}\right)$ as in Section 4 .

Case IIa: $w_{k}^{2} /\left(\sum_{j=k}^{n} w_{j}^{2}\right)>\epsilon^{2} / 144$ for all $k=1, \ldots, \ell$. As in Case IIa of Section 4 we let

$$
W=w_{\ell+1}^{2}+\cdots+w_{n}^{2},
$$

but now we set

$$
\eta^{\prime}=2 \sqrt{W \ln (8 / \epsilon)}
$$

(compare this with the setting of $\eta$ as $\sqrt{W \ln (4 / \epsilon)}$ of the earlier proof). 
We have that $|F(x)| \leq \eta^{\prime} / 2$ only if either $\left|w_{\ell+1} x_{\ell+1}+\cdots+w_{n} x_{n}\right| \geq \eta^{\prime} / 2$ or $\left|w_{1} x_{1}+\cdots+w_{\ell} x_{\ell}-\theta\right| \leq \eta^{\prime}$. As in the derivation of (4.3) the Hoeffding bound gives us

$$
\operatorname{Pr}\left[\left|w_{\ell+1} x_{\ell+1}+\cdots+w_{n} x_{n}\right| \geq \eta^{\prime} / 2\right] \leq \epsilon / 4 .
$$

It remains to bound $\operatorname{Pr}\left[\left|w_{1} x_{1}+\cdots+w_{\ell} x_{\ell}-\theta\right| \leq \eta^{\prime}\right]$ by $\epsilon / 4$; again reasoning as in the earlier section it suffices to show that

$$
\operatorname{Pr}\left[\left|w_{1} x_{1}+\cdots+w_{\ell} x_{\ell}-\theta\right| \leq(24 / \epsilon) \sqrt{2 \ln (8 / \epsilon)} w_{\ell}\right] \leq \epsilon / 4 .
$$

Comparing this with (4.4), we see that the two expressions differ only in constant factors. One can verify that the arguments of Case IIa in Section 4 (with suitably adjusted constants) also yield (6.6) as desired.

We thus have that $\operatorname{Pr}\left[|F(x)| \leq \eta^{\prime} / 2\right] \leq \epsilon / 2$. From the definitions of $\eta^{\prime}$ and $W$ we have that $\eta^{\prime} / 2 \geq \sqrt{W} \geq\left|w_{\ell+1}\right|$, so consequently

$$
\operatorname{Pr}\left[|F(x)| \leq\left|w_{\ell+1}\right|\right] \leq \epsilon / 2 .
$$

Now let us suppose that $\tau>\epsilon$. Reasoning as in Case I, we thus have that at least $\frac{\epsilon}{2} 2^{n}$ many points $x \in D$ have $|F(x)|>\left|w_{\ell+1}\right|$. The bound (6.4) now gives

$$
\left(|\theta|+\sum_{i=1}^{n}\left|w_{i}\right|\right) / M \geq \frac{2}{2^{n}} \cdot \frac{\epsilon}{2} 2^{n} \cdot\left|w_{\ell+1}\right|
$$

which is equivalent to

$$
M \leq \frac{|\theta|+\sum\left|w_{i}\right|}{\epsilon\left|w_{\ell+1}\right|}
$$

Since $|\theta| \leq \sum\left|w_{i}\right|$, we have that

$$
\begin{aligned}
v M \leq \frac{2}{\epsilon} \cdot \frac{\sum_{i=1}^{n}\left|w_{i}\right|}{\left|w_{\ell+1}\right|} & \leq \frac{2}{\epsilon}\left(\frac{\ell}{\left|w_{\ell+1}\right|}+\frac{\sum_{i=\ell+1}^{n}\left|w_{i}\right|}{\left|w_{\ell+1}\right|}\right) \\
& \leq \frac{2}{\epsilon}\left(\frac{\ell}{\left|w_{\ell+1}\right|}+n\right) \\
& \leq \frac{2}{\epsilon}(\ell \cdot(\ell+1) !(n+1)+n)
\end{aligned}
$$

where the second inequality holds since each of $\left|w_{1}\right|, \ldots,\left|w_{\ell}\right|$ is at most 1 , the third inequality holds since each of $\left|w_{\ell+1}\right|, \ldots,\left|w_{n}\right|$ is at most $\left|w_{\ell+1}\right|$, and the fourth inequality follows from Theorem 6.5. But recalling that $\ell=\tilde{O}\left(1 / \epsilon^{2}\right)$, this upper bound on $M$ contradicts the fact that $M=n \cdot 2^{\tilde{O}\left(1 / \epsilon^{2}\right)}$ (for a suitable 
choice of the hidden polylogarithmic factor in the exponent of the definition of $M)$.

Case IIb: $w_{k}^{2} /\left(\sum_{j=k}^{n} w_{j}^{2}\right) \leq \epsilon^{2} / 144$ for some $k \in\{1, \ldots, \ell\}$. For each $i=$ $1, \ldots, n$ let $v_{i}$ denote $w_{i} /\left|w_{k}\right|$, so we have $1=\left|v_{k}\right| \geq\left|v_{k+1}\right| \geq \cdots \geq v_{n}$. Using Theorem 2.2 with $\lambda=1$, we have that for all $\tau \in \mathbf{R}$,

$$
\begin{aligned}
\operatorname{Pr}_{x_{k}, \ldots, x_{n}}\left[\left|\sum_{j=k}^{n} w_{j} x_{j}-\tau\right| w_{k}|| \leq\left|w_{k}\right|\right] & =\operatorname{Pr}_{x_{k}, \ldots, x_{n}}\left[\left|v_{k} x_{k}+\cdots+v_{n} x_{n}-\tau\right| \leq 1\right] \\
& \leq 6 / \sqrt{v_{k}^{2}+\cdots+v_{n}^{2}} \\
& =6\left|w_{k}\right| / \sqrt{w_{k}^{2}+\cdots+w_{n}^{2}} \leq \epsilon / 2
\end{aligned}
$$

where the last inequality holds since we are in Case IIb. It follows that for any $\theta \in \mathbf{R}$ we have

$$
\operatorname{Pr}_{x_{1}, \ldots, x_{n}}\left[\left|w_{1} x_{1}+\cdots+w_{n} x_{n}-\theta\right| \leq\left|w_{k}\right|\right]=\operatorname{Pr}_{x_{1}, \ldots, x_{n}}\left[|F(x)| \leq\left|w_{k}\right|\right] \leq \epsilon / 2 .
$$

Now an entirely similar argument to that given from (6.7) through the end of Case IIa shows that as in that case, we must have $\tau \leq \epsilon$. This concludes the analysis of all cases, so Theorem 1.2 is proved.

6.2. Proof of Theorem 6.5. We first consider the case in which $f(x)=$ $f(-x)$ for all $x \in\{-1,1\}^{n}$, i.e., $f$ can be represented with a threshold of zero. Once we have the result for such $f$ we will use it to prove the result for general $f$.

Let $\operatorname{sgn}\left(w_{1} x_{1}+\cdots+w_{n} x_{n}\right)$ be a representation for $f$ which satisfies the conditions

1. $\operatorname{sgn}(w \cdot x)=f(x)$ for each $x \in\{-1,1\}^{n}$.

2. $|w \cdot x| \geq 1$ for each $x \in\{-1,1\}^{n}$.

3. Among all vectors in $\mathbf{R}^{n}$ which satisfy conditions (1) and (2) above, $w$ maximizes the number of $x \in\{-1,1\}^{n}$ which have $|w \cdot x|=1$. If there is more than one such $w$, choose one arbitrarily.

The argument in Section 3 of Håstad (1994) now implies that there is a set $x^{(1)}, \ldots, x^{(n)}$ of $n$ elements of $\{-1,1\}^{n}$ such that the coefficients $w_{1}, \ldots, w_{n}$ are determined as the unique solution to the system of equations

$$
v_{1} x_{1}^{(i)}+\cdots+v_{n} x_{n}^{(i)}=f\left(x^{(i)}\right) \quad \text { for } \quad i=1, \ldots, n .
$$


This is a system of $n$ equations in the variables $v_{1}, \ldots, v_{n}$ where each coefficient is \pm 1 and the right-hand side of each equation, $f\left(x^{(i)}\right)$, is also \pm 1 . Recall that $f$ depends on all $n$ variables and consequently we have that each $w_{i}$ - and in particular $w_{n}$ - is nonzero. Using this fact it is not difficult to see that the above system of equations is equivalent to the following system of $n$ equations in $v_{1}, \ldots, v_{n}$ :

$$
\begin{aligned}
f\left(x^{(1)}\right)\left(v_{1} x_{1}^{(1)}+\cdots+v_{n} x_{n}^{(1)}\right) & =f\left(x^{(i)}\right)\left(v_{1} x_{1}^{(i)}+\cdots+v_{n} x_{n}^{(i)}\right) \text { for } i=2, \ldots, n, \\
v_{n} & =w_{n} .
\end{aligned}
$$

(The first $n-1$ homogeneous equations above have a one-dimensional set of solutions, and the final equation $v_{n}=w_{n}$ specifies the unique correct solution to the whole system.) Each of these first $n-1$ equations has no constant term and (dividing by two and rearranging) can be rewritten as $v \cdot y^{(i)}=0$, where $y^{(i)}$ is a vector whose entries are all $-1,0$ or 1 . So we have that $w_{1}, \ldots, w_{n}$ is the solution to the system of equations

$$
Y v=b
$$

where $Y$ is a nonsingular $n \times n$ matrix with $\{-1,0,1\}$ entries where the last

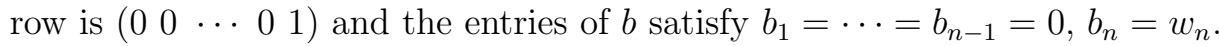

We assume that $\left|w_{1}\right| \geq\left|w_{2}\right| \geq \cdots \geq\left|w_{n}\right|$, and now show that $\left|w_{k}\right|$ must be somewhat large compared with $\left|w_{1}\right|$.

After possibly reordering the first $n-1$ equations, we can find a linear combination of the first $k-1$ equations such that the only nonzero coefficient among $v_{1}, \ldots, v_{k-1}$ belongs to $v_{1}$, i.e., an equation of the form

$$
v_{1}=\sum_{j=k}^{n} a_{j} v_{j} .
$$

Using Cramer's Rule and the fact that any $(k-1) \times(k-1)$ matrix with entries in $\{-1,0,1\}$ has determinant at most $(k-1)$ !, it is not hard to show that an equality in the form of (6.9) must exist where each $\left|a_{j}\right| \leq(k-1)$ !. But now if $\left|w_{k}\right|<\frac{\left|w_{1}\right|}{(k-1) !(n-k+1)}$, then it is impossible for $w$ to satisfy (6.9) since the right-hand side must be too small. This proves that

$$
\left|w_{k}\right| \geq \frac{\left|w_{1}\right|}{(k-1) !(n-k+1)} \geq \frac{\left|w_{1}\right|}{(k-1) ! n},
$$

so we are done in the zero-threshold case. 
We can treat the case where $f$ has a nonzero threshold by considering the function $g:\{-1,1\}^{n+1} \rightarrow\{-1,1\}$ which has zero threshold but an $(n+1)$-st weight which is the threshold of $f$. The argument for the zero-threshold case now shows that $g$ has a representation $\operatorname{sgn}\left(w_{1} x_{1}+\cdots+w_{n} x_{n}+w_{n+1} x_{n+1}\right)$ with $\left|w_{1}\right| \geq \cdots \geq\left|w_{n+1}\right|$ and $\left|w_{k}\right| \geq \frac{\left|w_{1}\right|}{(k-1) !(n+1)}$; note that one of these $w_{i}$ weights actually corresponds to the threshold of the original LTF $f$. If $w_{1}$ is the threshold then $w_{2}$ is actually the largest weight of $f$ in magnitude and we have $\left|w_{k}\right| \geq \frac{\left|w_{2}\right|}{(k-1) !(n+1)}$. If $w_{r}$ is the threshold for some $r>1$ then $w_{1}$ is indeed the largest of $f$ 's weights. In this case, for $k<r$ we have that $f$ 's $k$-th biggest weight is $w_{k}$ which satisfies $\left|w_{k}\right| \geq \frac{\left|w_{1}\right|}{(k-1) !(n+1)}$, whereas for $k>r$ we have that $f$ 's $k$-th biggest weight is $w_{k+1}$ which satisfies $\left|w_{k+1}\right| \geq \frac{\left|w_{1}\right|}{k !(n+1)}$. So in every case the magnitude of the $k$-th biggest weight is at least $\frac{1}{k !(n+1)}$ times the magnitude of the biggest weight, and Theorem 6.5 is proved.

6.3. Lower bounds on required accuracy for Chow parameter estimation. In this section we sketch a simple argument which shows that no variant of Theorem 1.2 in which the bound on $|\hat{g}(S)-\hat{f}(S)|$ is $1 / o(\sqrt{n})$ (as a function of $n$ ) can be true. Suppose to the contrary that Theorem 1.2 held with a bound of the form $1 /(o(\sqrt{n} \cdot \kappa(\epsilon))$ for some function $\kappa$ that depends only on $\epsilon$. If we fix $\epsilon$ to be a constant such as $1 / 10$, the bound is simply $1 / o(\sqrt{n})$. It is well known, and easy to verify, that the majority function on $n$ variables has all its Chow parameters $1 / \Theta(\sqrt{n})$. If accuracy $1 / o(\sqrt{n})$ were sufficient, then for sufficiently large $n$ we could take $g$ to be any function with Chow parameters all 0 , such as the parity function on $n$ variables; but the majority function is $(1 / 2-o(1))$-far from the parity function on $n$ variables.

\section{Conclusion}

We hope that Theorem 1.1 may find a range of applications in future work. In computational learning theory, low-weight linear threshold functions are known to be "nice" in several senses; our results suggest that similar properties might sometimes hold for arbitrary linear threshold functions as well. As one example, simple and efficient algorithms are known which can learn low-weight linear threshold functions under noise rates at which no efficient algorithms are known for learning arbitrary linear threshold functions. Can our results (which can be viewed as stating that every linear threshold function is "close to" a low-weight linear threshold function) be used to learn arbitrary linear threshold functions in the presence of higher noise rates?

More concretely, an obvious direction for future work is to improve the 
asymptotic dependence on $\epsilon$ in our results. As Goldberg (2001) and Servedio (2004) have observed, Håstad's construction of a linear threshold function which requires integer weights of size $2^{\Omega(n \log n)}$ implies that in general an $\epsilon$ approximating LTF for an arbitrary LTF $f$ may require integer weights of size $(1 / \epsilon)^{\Omega(\log \log (1 / \epsilon))}$. While this means that it is impossible to obtain an analogue of Theorem 1.1 with a poly $(1 / \epsilon)$ dependence on $\epsilon$, it may well be possible to improve the current $2^{\tilde{O}\left(1 / \epsilon^{2}\right)}$ dependence.

Another goal is to obtain stronger bounds on the accuracy which is required in the Chow parameters in order to specify an arbitrary linear threshold function $f$ to accuracy $\epsilon$. Can the gap between our $1 / O(n)$ bound and the $1 / \Omega(\sqrt{n / \log n})$ bound given in Section 6.3 be closed?

A final ambitious goal is to investigate a distributional version of the problem, in which the approximation is measured with respect to an arbitrary probability distribution. If positive results could be obtained in this setting, they might lead to interesting consequences for distribution-independent learning of linear threshold functions.

\section{Acknowledgements}

This research was partially supported by NSF CAREER award CCF-0347282, by NSF award CCF-0523664, and by a Sloan Foundation Fellowship.

The author thanks Johan Håstad for his kind permission to include the proof of Theorem 6.5 in this paper, and thanks Adam Klivans for many stimulating discussions on these topics without which this paper would never have been written. The author also thanks the anonymous journal referees who made many helpful suggestions, in particular pointing out the simple argument used in Section 6.3.

\section{References}

S. Ben-David \& E. Dichterman (1994). Learnability with Restricted Focus of Attention guarantees Noise-Tolerance. In Proceedings of the 5th International Workshop on Algorithmic Learning Theory, 248-259.

S. Ben-DAVid \& E. Dichterman (1998). Learning with restricted focus of attention. Journal of Computer and System Sciences 56(3), 277-298.

A. Birkendorf, E. Dichterman, J. Jackson, N. Klasner \& H. U. Simon (1998). On restricted-focus-of-attention learnability of Boolean functions. Machine Learning 30, 89-123. 
H. Block (1962). The Perceptron: a model for brain functioning. Reviews of Modern Physics 34, 123-135.

J. BRUCK (1990). Harmonic analysis of polynomial threshold functions. SIAM Journal on Discrete Mathematics 3(2), 168-177.

C. K. Chow (1961). On the characterization of threshold functions. In Proceedings of the Symposium on Switching Circuit Theory and Logical Design, 34-38.

M. Dertouzos (1965). Threshold logic: a synthesis approach. MIT Press, Cambridge, MA.

M. Dyen (2003). Approximate Counting by Dynamic Programming. In Proceedings of the 35th Annual Symposium on Theory of Computing (STOC), 693-699.

M. Dyer, A. Frieze, K. Rannan, A. Kapoor, L. Perkovic \& U. Vazirani (1993). A mildly exponential time algorithm for approximating the number of solutions to a multidimensional knapsack problem. Combinatorics, Probability and Computing 2, 271-284.

Y. FReUnd (1995). Boosting a weak learning algorithm by majority. Information and Computation 121(2), 256-285.

Y. Freund \& R. Schapire (1997). A decision-theoretic generalization of on-line learning and an application to boosting. Journal of Computer and System Sciences 55(1), 119-139.

P. GoldBerg (2001). Estimating a Boolean perceptron from its average satisfying assignment: Aa bound on the precision required. In Proceedings of the Fourteenth Annual Conference on Computational Learning Theory, 116-127.

M. Goldmann, J. HÅstad \& A. Razborov (1992). Majority gates vs. general weighted threshold gates. Computational Complexity 2, 277-300.

M. Goldmann \& M. Karpinski (1998). Simulating threshold circuits by majority circuits. SIAM Journal on Computing 27(1), 230-246.

A. Hajnal, W. Maass, P. Pudlak, M. Szegedy \& G. Turan (1993). Threshold circuits of bounded depth. Journal of Computer and System Sciences 46, 129-154.

J. HÅstad (1994). On the size of weights for threshold gates. SIAM Journal on Discrete Mathematics 7(3), 484-492.

J. HÅstad (2005). Personal communication. 
T. Hofmeister (1996). A Note on the Simulation of Exponential Threshold Weights. In Computing and Combinatorics, Second Annual International Conference (COCOON), 136-141.

J. Hong (1987). On connectionist models. Technical Report 87-012, Dept. of Computer Science, University of Chicago.

S. T. Hu (1965). Threshold Logic. University of California Press.

M. Jerrum \& A. Sinclair (1997). The Markov Chain Monte Carlo method: an approach to approximate counting and integration, 482-520. PWS Publishing.

R. Kannan (1994). Markov chains and polynomial time algorithms. In Proceedings of the 35th Symposium on Foundations of Computer Science, 656-671.

P. Kaszerman (1963). A geometric test-synthesis procedure for a threshold device. Information and Control 6(4), 381-398.

A. Klivans, R. O'Donnell \& R. Servedio (2004). Learning intersections and thresholds of halfspaces. Journal of Computer \& System Sciences 68(4), 808-840. Preliminary version in Proc. of FOCS'02.

A. Klivans \& R. Servedio (2001). Learning DNF in time $2^{\tilde{O}\left(n^{1 / 3}\right)}$. In Proceedings of the Thirty-Third Annual Symposium on Theory of Computing, 258-265.

N. Littlestone (1988). Learning quickly when irrelevant attributes abound: a new linear-threshold algorithm. Machine Learning 2, 285-318.

N. Littlestone (1991). Redundant noisy attributes, attribute errors, and linearthreshold learning using Winnow. In Proceedings of the Fourth Annual Workshop on Computational Learning Theory, 147-156.

M. Luby \& B. Velickovic (1996). On deterministic approximation of DNF. Algorithmica 16(4/5), 415-433.

W. MaAss \& G. Turan (1994). How fast can a threshold gate learn?. In Computational Learning Theory and Natural Learning Systems: Volume I: Constraints and Prospects, S. Hanson, G. Drastal, and R. Rivest, eds., 381-414. MIT Press.

B. Morris \& A. Sinclair (1999). Random walks on truncated cubes and sampling 0-1 Knapsack Solutions (Preliminary Version). In Proceedings of the 40th Annual Symposium on Foundations of Computer Science (FOCS), 230-240.

S. Muroga (1971). Threshold logic and its applications. Wiley-Interscience, New York. 
S. Muroga, I. Toda \& S. TAKAsu (1961). Theory of majority switching elements. J. Franklin Institute 271, 376-418.

A. Novikoff (1962). On convergence proofs on perceptrons. In Proceedings of the Symposium on Mathematical Theory of Automata, volume XII, 615-622.

P. Orponen (1992). Neural networks and complexity theory. In Proceedings of the 17th International Symposium on Mathematical Foundations of Computer Science, $50-61$.

V. V. Petrov (1995). Limit theorems of probability theory. Oxford Science Publications, Oxford, England.

P. Raghavan (1988). Learning in threshold networks. In First Workshop on Computational Learning Theory, 19-27.

A. Razborov (1992). On Small Depth Threshold Circuits. In Proceedings of the Third Scandinavian Workshop on Algorithm Theory (SWAT), 42-52.

R. SERvedio (2004). Monotone Boolean formulas can approximate monotone linear threshold functions. Discrete Applied Mathematics 142(1-3), 181-187.

J. Shawe-TAylor \& N. CRistianini (2000). An introduction to support vector machines. Cambridge University Press.

L. TREvisAn (2004). A note on approximate counting for $k$-DNF. In Proceedings of the Eighth International Workshop on Randomization and Computation, 417-426.

L. VALiAnt (1984). Short monotone formulae for the majority function. Journal of Algorithms 5, 363-366.

R. O. Winder (1971). Chow parameters in threshold logic. Journal of the ACM 18(2), 265-289.

Manuscript received 10 September 2006

Rocco A. SERVEdio

Department of Computer Science

Columbia University

1214 Amsterdam Avenue, Mailcode 0401

New York, NY 10027-7003, USA

rocco@cs.columbia.edu 